\title{
La evaluación basada en el aprendizaje desde la experiencia
}

\section{Evaluation based on learning from the experience}

\section{Diana Marcela Cortes Carmona ${ }^{1}$}

\section{Catalina Gallego Tobón ${ }^{2}$}

Yancelly Gómez Parra ${ }^{3}$

\section{Nini Johana Mejía López ${ }^{4}$}

Cindy Paola Panesso 5

Universidad de San Buenaventura

\begin{abstract}
$\overline{11}{ }^{1}$ Licenciada en Educación Preescolar, Especialista en Gerencia Educativa, Magister en Educación de la Universidad de San Buenaventura. Docente de la Licenciatura en Educación Infantil de la Universidad de San Buenaventura, diamarce1113@hotmail.com Orcid 0000000150948463

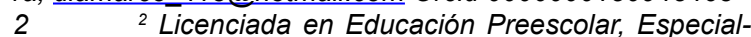
ista en Gerencia Educativa, Magister en Educación de la Universidad de San Buenaventura. Docente investigadora de la Licenciatura en Educación Infantil de la Universidad de San Buenaventura. catagallegot@gmail.com Orcid 0000000176720862

$3{ }^{3}$ Licenciada en Educación Preescolar, especialista en gerencia Educativa, Magíster en Educación, docente investigadora de la Licenciatura en Educación infantil de la universidad de San Buenaventura Medellín. yancelygomezparra@gmail.com Orcid 000000025465438X

$4{ }^{4}$ Licenciada en Educación Preescolar, Candidata a Magister en Ciencias de la Educación de la Universidad de San Buenaventura Medellín. niniyo98@gmail.com Orcid 0000000266974612

$5 \quad$ Licenciada en Educación Preescolar, Magíster en Educación de la Universidad San Buenaventura. Docente de la Licenciatura en Educación Infantil de la universidad San Buenaventura.
\end{abstract}

Paopanesso2013@gmail.com Orcid 000000032997141X

\section{RESUMEN}

El presente artículo de reflexión se plantea como objetivo contribuir en la resignificación del concepto de evaluación, considerando sus distintas implicaciones y las diferentes concepciones que se articulan a dicho proceso, vinculando distintos actores que participan en el quehacer educativo, para finalmente considerar la pertinencia de los procesos evaluativos en educación inicial como un aprendizaje desde la experiencia. De acuerdo a dicho objetivo, se realizó un recorrido a través de cuatro apartados temáticos, 1.) ¿Qué es la evaluación?: propone un acercamiento a las definiciones de evaluación; 2.) Desde la medición a procesos de aprendizaje: presenta la evaluación como herramienta de medición y 
se vislumbra la posibilidad de una evaluación para la reflexión y generación de aprendizajes significativos; 3.) El rol del maestro de hoy frente al proceso evaluativo: se discute y argumenta las implicaciones del significado de evaluación en la praxis docente, y 4.) La evaluación en la educación inicial: se consideran la aplicación de los argumentos anteriores en las primeras etapas educativas.

Finalmente, en las conclusiones se aboga por una evaluación que considere el aprendizaje desde la experiencia, lo cual requiere poner el acento en una aproximación cualitativa en los procesos evaluativos.

PALABRAS CLAVE: evaluación, experiencia, medición, aprendizaje significativo

\section{ABSTRACT}

This article of reflection aims to contribute to the resignification of the concept of evaluation, considering its different implications and the different conceptions that are articulated to said process, linking different actors involved in the educational task, to finally consider the relevance of the Evaluative processes in initial education as learning from experience. According to this objective, a tour was carried out through four thematic sections, 1.) What is the evaluation?: proposes an approach to the definitions of evaluation; 2.) From the measurement to learning processes: it presents the evaluation as a measurement tool and the possibility of an evaluation for the reflection and generation of significant learning is envisioned; 3.) The role of today's teacher in the evaluation: the implications of the meaning of evaluation in teaching praxis are discussed and argued, and 4.) Evaluation in early education: the application of the previous arguments in the Early educational stages

Finally, the conclusions advocate an evaluation that considers learning from experience, which requires emphasizing a qualitative approach in the evaluation processes.

KEY WORDS: evaluation, experience, measurement, meaningful learning

\section{INTRODUCCIÓN .}

El concepto de evaluación ha sido objeto de diversas definiciones y connotaciones, lo cual ha posibilitado diferentes interpretaciones con sus debidas implicaciones en la práctica educativa.

Es evidente a través del rastreo bibliográfico un sinnúmero de definiciones, e incluso la omisión de la conceptualización científica, ya sea porque los escritores asumen un conocimiento previo del lector, porque hay confusión en el tema o falta de claridad. Lo realmente peculiar es que esta ambigüedad conceptual ha derivado en formas diversas en cuanto al significado y formas de evaluar por parte del docente, considerando especialmente la evaluación como un método de control sobre los estudiantes, así como un acto de requerimiento administrativo.

Por otro lado, la evaluación se ha convertido en un procedimiento que solo permite medir y clasificar de acuerdo a unos resultados implícitamente programáticos, tratando de homogeneizar los procesos en el aula.

De acuerdo a lo anterior, se hace necesario resignificar los métodos y procedimientos de evaluación, lo cual debe partir de una tarea reflexiva previa sobre el concepto y su finalidad, asumiendo que es el docente el encargado de vincular el trabajo de enseñanza y aprendizaje con la experiencia ${ }^{6}$ significativa en el aula.

En tal sentido, es necesario que el maestro de hoy se cuestione frente a sus métodos de

$6 \quad$ Cuando se hace referencia al concepto experiencia, se parte de la noción del aprendizaje desde la experiencia que tienen los estudiantes en el aula a partir de su cotidianidad, la cual es articulada al proceso formativo y al proceso de aprendizaje, porque sólo desde la experiencia, de lo real y la manipulación se puede hablar de lo significativo. 
enseñanza, partiendo del principio de que su praxis genera determinados aprendizajes en un proceso de exploración conjunta con sus estudiantes, en lugar de asumir el rol de conocedor de un todo donde solo se escucha su voz autorizada.

El objetivo de este trabajo es contribuir teóricamente en la resignificación del concepto de evaluación, considerando sus distintas implicaciones y las diferentes concepciones que se articulan con dicho proceso. Para tal fin se analizan distintos actores y escenarios que participan en el quehacer educativo y se considera la pertinencia de los procesos evaluativos en educación inicial como un aprendizaje desde la experiencia.

En este sentido, se da a conocer el recorrido del texto conformado por cuatro apartados, ¿Qué es la evaluación? en el cual se propone un acercamiento a sus definiciones; Desde la medición a procesos de aprendizaje, en este punto se considera la evaluación como herramienta de medición y se visibiliza la posibilidad de una evaluación para la reflexión y generación de aprendizajes significativos; El rol del maestro de hoy frente al proceso evaluativo, en este apartado se discute y argumenta las implicaciones del significado de evaluación en la praxis docente, y finalmente La evaluación en la educación inicial, un proceso articulador,, en el cual se consideran la aplicación de los argumentos anteriores en las primeras etapas educativas.

Cabe mencionar que los apartados desarrollados no pretenden ser concluyentes sobre un proceso que está llamado a ser debatido, sino que, por el contrario, son tentativos, reflexivos y críticos frente a la praxis docente orientada a una evaluación articulada con el aprendizaje desde la experiencia.

\section{1. ¿QUÉ ES LA EVALUACIÓN?}

La evaluación se ha convertido en un proceso fundamental para asuntos relacionados con la calidad educativa. Se evalúa a los estudiantes, se evalúa a los docentes y se evalúan las instituciones, esperando obtener los mejores resultados, y de lo contrario, implementar planes de acción para su optimización.

Específicamente en el ámbito de los procesos de aprendizaje, la evaluación permite hacer un seguimiento a los estudiantes indicando el nivel de logro y el avance que cada uno presenta en relación a determinados contenidos. Si bien desde las políticas educativas colombianas se proponen unas orientaciones frente al proceso evaluativo y que este se centre en los estudiantes respetando la individualidad y las necesidades que a nivel de aula se presentan, en realidad, la evaluación se plantea como un proceso uniforme y homogéneo, tal y como se expresa en el Decreto 1290, por el cual se reglamenta la evaluación del aprendizaje y promoción de los estudiantes de los niveles de educación básica y media, dispuesto por el Ministerio de Educación Nacional (2009).

Para dar continuidad al tema que ocupa, es importante atender el artículo 3 del Decreto antes mencionado, donde se plantea los siguiente:

Artículo 3. Propósitos de la evaluación institucional de los estudiantes. Son propósitos de la evaluación de los estudiantes en el ámbito institucional:

1. Identificar las características personales, intereses, ritmos de desarrollo y estilos de aprendizaje del estudiante para valorar sus avances.

2. Proporcionar información básica para consolidar o reorientar los procesos educativos relacionados con el desarrollo integral del estudiante. 
3. Suministrar información que permita implementar estrategias pedagógicas para apoyar a los estudiantes que presenten debilidades y desempeños superiores en su proceso formativo.

4. Determinar la promoción de estudiantes.

5. Aportar información para el ajuste e implementación del plan de mejoramiento institucional.

De lo anterior se desprende que el proceso evaluativo no solo está dirigido a los procesos de aprendizaje individuales, sino que están articulados a las políticas pedagógicas y de calidad institucional. En tal sentido, cuando hay preocupación a nivel institucional frente al proceso evaluativo, internamente todo un equipo de trabajo estaría estructurando un plan integrado que permita incidir positivamente en los resultados de la evaluación, ya que éstos hacen parte de la calidad educativa y formación de los estudiantes.

Ahora bien, es necesario reflexionar acerca de cómo lograr que desde la experiencia individual y el compartir colectivo en el aula se establezca el punto de partida para enseñar y aprender, y que en relación a esto se construyan los indicadores de calidad. De allí la necesidad de centrar la mirada en los estudiantes para resignificar el proceso de enseñanza-aprendizaje, la práctica docente, la metodología, la didáctica, los contenidos y la pertinencia en ese devenir del proceso educativo. Tal resignificación pensada desde la reflexión del docente daría otro sentido menos temido y angustiante por parte de los estudiantes frente al concepto de evaluación.

\section{DESDE LA MEDICIÓN A PROCESOS DE APRENDIZAJE}

Después de lo tratado hasta aquí, es importante precisar que este trabajo reflexivo intenta contrastar; por un lado, la evaluación como herramienta de medición y por otro lado, visibilizar la posibilidad de una evaluación más desde la reflexión y generación de aprendizajes significativos.

La evaluación no es una práctica reciente en el ámbito educativo. Ya desde la edad media se introducen en las dinámicas escolares exámenes como herramientas de medición, pero no consideraban una visión holística de la evaluación que integrara a todos los elementos participantes. Es en el siglo XIX, con el movimiento reformista educativo, cuando se ve la necesidad de generar acciones en donde la evaluación tenga un lugar dentro del currículo (Tamayo, Niño, Cardozo y Bejarano, 2017).

Desde la concepción de rendimiento económico, vinculado a la calidad del trabajo, Taylor aparece como el padre de la evaluación educativa, desde su propuesta del método sistémico por objetivos, generando una perspectiva de evaluación enfocada a cuestiones como ¿Qué evaluar? ¿Cómo evaluar?, ¿Cuándo evaluar?, ¿Con qué evaluar? (Tamayo y otros, ob.cit). Esto permite que se empiecen a generar cuidadosamente análisis frente a los propósitos de la evaluación en las prácticas pedagógicas.

En la década de los noventa y gracias al fenómeno de la globalización, se inicia una búsqueda al mejoramiento de la calidad educativa, lo que implica una reforma en los sistemas educativos y las exigencias del mismo, lo cual permite la incorporación de las pruebas externas de medición de calidad. Para Jurado (2009) este sistema de evaluación externa ha permitido que, en los países de América Latina, la evaluación constituya una herramienta para la orientación de los procesos educativos y el establecimiento de políticas públicas por parte del Estado para mejorar la educación. 
Es interesante resaltar que a pesar de todos los cambios estructurales frente a la concepción de evaluación, un nuevo concepto de calidad entra a circular en el ámbito educativo, la calidad como el resultado y análisis de la aplicación de unas pruebas estandarizadas elaboradas por instituciones encargadas para ello, lo que ha venido arrojando información estadística y comparativa entre países (OCDE, 2018). Si bien es importante como ejercicio, no es conveniente quedar en análisis meramente estadísticos y de resultados de una aplicación de pruebas generales para todas las comunidades a nivel nacional como instrumento de valoración de los niveles de aprendizaje.

Es gracias a esto que se propone re pensar en la paradoja en la cuales se sustentan las prácticas pedagógicas hoy en día; esta paradoja es el poder responder a unas pruebas estandarizadas y a la vez proporcionar herramientas significativas a unos sujetos que están en procesos de formación y cambio constante, lo que genera en las dinámicas institucionales algunas tensiones y preocupaciones frente al tema evaluativo.

Lo anterior permite afirmar que la evaluación como práctica pedagógica y didáctica es un proceso tan complejo que no debe ser reducida a la medición de resultados, sino que, por el contrario, se vincula a prácticas, epistemologías y diseños curriculares específicos (Tamayo y otros, Ob.cit.). Dicho planteamiento coloca al maestro y al alumno en un acto de interrelación y en un rol activo y protagónico del proceso de formación, lo que posibilita a un acercamiento al concepto a la evaluación como proceso de aprendizaje desde la experiencia.

Para hablar de la evaluación desde una particularidad del sujeto, es indispensable considerar el contexto, pues será el reconocimiento del mismo el que permita al docente generar comprensiones y entender realidades de los sujetos que interactúan en espacios escolares de formación. No debe olvidarse, tal y como ya se ha venido exponiendo, que la escuela es el escenario en el cual más tensiones se generan alrededor del tema de evaluación, puesto que el ser humano es parte y se transforma con el entorno en el cual interactúa. (Ortiz, 2015)

El reconocimiento del contexto permitirá a los docentes y demás agentes educativos miradas amplias y heterogéneas de la evaluación, ya que si concebimos el aprendizaje como un proceso sistémico e inacabado, resulta lógico pensar en una evaluación en esa misma perspectiva.

Según Maturana, citado por Ortiz "la medición y evaluación de los conocimientos de los niños y niñas debe ser vivida por ellos como una oportunidad para resolver dificultades y no como una amenaza. El acto de confianza que tal actitud implica amplía el espacio de aprendizaje de los niños y niñas, y facilita su orientación por los profesores y las profesoras" (Leal Ortiz, 2014). Esto significa una visión más holística de la evaluación, en la cual se resignifiquen las prácticas pedagógicas a la luz de las necesidades de los estudiantes.

La evaluación así concebida "es un proceso de comunicación y diálogo permanente y respetuoso que tiene en cuenta los intereses, pre concepciones y contextos de cada estudiante, pero también los saberes" (Tamayo y otros, ob. cit. p. 69). Esto implica que el docente no solo debe asumir juicios de valor frente a los conocimientos de los estudiantes, sino reconocer un contexto, un espacio y unos sujetos activos de su propio proceso formativo, asumiendo la evaluación como un proceso nunca exclusivo, ni repetitivo o mecánico.

Durante el recorrido temático de este texto se ha venido hablando sobre el concepto de evaluación y su mirada desde la medición, pero no debe olvidarse que también se ha hecho referencia a que, gracias a procesos reflexivos, 
se han generado otros diálogos diferentes sobre este tema. Es por ello que en este punto es importante traer a colación los procesos de retroalimentación dentro de la evaluación, como una estrategia inherente a la concepción de evaluación en el siglo XXI, orientada a la formación de los sujetos a fin de dar respuesta a los diferentes retos educativos.

La retroalimentación entendida como un intercambio entre docente y estudiante favorece a su vez la motivación, y dota de sentido los procesos de enseñanza y aprendizaje. El Ministerio de Educación Nacional en Colombia afirma

\begin{abstract}
"Una evaluación adecuada y transparente hace explícitas sus reglas y objetivos en relación con los desempeños que evalúa y permite abordar estrategias para los aprendizajes que se dificultan, teniendo en cuenta los intereses y contextos de cada uno. Es importante que su práctica de aula ofrezca una retroalimentación positiva, que fortalezca la autoestima y empodere a los estudiantes para seguir mejorando" (MEN 2008, s/p)
\end{abstract}

Dicho lo anterior, se evidencia cómo la evaluación de los aprendizajes ha generado diferentes discursos y tensiones, las cuales van configurando día a día las prácticas pedagógicas de los docentes, sin dejar de reconocer un contexto y unas necesidades particulares en los estudiantes. Es por ello que en el próximo apartado se plantea el papel del docente en todo el proceso evaluativo y sobre todo, como sujeto activo en el contexto de la escuela.

\section{EL ROL DEL MAESTRO DE HOY FRENTE AL PROCESO EVALUATIVO}

El rol del maestro frente a los procesos evaluativos debe romper con el esquema tradicional de evaluar para que los estudiantes respondan lo que el maestro quiere escuchar o leer. Es menester que en la práctica docente haya una reflexión sobre qué es la evaluación y el sentido por la cual se implementa, y de esa manera aplicarla desde una postura realmente formativa. En otras palabras, es importante el rol del maestro en el proceso de enseñanzaaprendizaje y cómo interviene, orienta y acompaña para que el aprendizaje no sea un logro de la mayoría, sino de todos.

El maestro está sujeto a promover espacios de aprendizaje basados en la experiencia y realidad de los estudiantes, dando sentido, articulación y coherencia de lo que planea en cada encuentro. De esta manera será más eficaz que los estudiantes asimilen y hagan conexión de nuevos contenidos con los saberes previos dentro de su proceso académico. Sería importante que antes de toda evaluación se dé espacio al diálogo, la participación, intercambio de ideas, a fin de cumplir con los objetivos propuestos.

La evaluación como elemento regular del trabajo en el aula, es una herramienta para promover el aprendizaje efectivo, la pertinencia de la enseñanza, la comprensión de las metas del aprendizaje y la motivación del estudiante. Las experiencias exitosas de la evaluación en el aula, muestran la importancia de la evaluación permanente, la participación activa y la autoevaluación del estudiante, la retroalimentación asertiva del docente y la confianza en el mejoramiento. El reto de los establecimientos educativos privados es establecer un sistema de evaluación coherente con el PEI, contextualizado e integrado al proceso de promoción del estudiante. (MEN, 2009, s/p)

Otro aspecto importante que debe asumir el maestro, es cómo se abordan las competencias, 
concepto que también ha sido mal desarrollado. No se trata de competencias para competir entre estudiantes ni clasificar el mejor desempeño, pues siempre habrá diferencias desde la individualidad y la diversidad; por el contrario, se trata de ser competentes para la vida, desde lo personal, social y laboral que facilitan el desempeño flexible y con sentido de una actividad en contextos relativamente nuevos y retadores (MEN, 2009)

Las competencias surgen a través de los cuatro pilares de la educación propuestos por Delors (1996), "aprender a conocer, aprender a hacer, aprender a vivir juntos y aprender a ser", los cuales serán fundamentales en la vida de las personas. Desde estos pilares los estudiantes podrán acceder al conocimiento desde una visión pedagógica integral que promueva estrategias facilitadoras del aprendizaje, que, a su vez, fortalecen el proceso y resultado evaluativo.

\section{LA EVALUACIÓN EN LA EDUCACIÓN INICIAL, UN PROCESO ARTICULADOR}

Es importante comprender que la evaluación no es un asunto que implica únicamente la medición de los aprendizajes, ni tampoco es inherente exclusivamente a práctica educativa. Evidentemente, de acuerdo a las diferentes etapas educativas, los procesos de evaluación pueden variar e incluso tener mayor o menor grado de estructuración.

En este apartado se hará especial referencia a la evaluación en la educación inicial. En esta etapa educativa, se hace necesario pensar la evaluación en términos de acompañamiento, seguimiento, control, pero sobre todo comprenderla en clave de transiciones y que estas transiciones tienen en cuenta todas las dimensiones del desarrollo del ser humano, tomando en cuenta que en este período evolutivo se generan importantes transformaciones no solo físicas sino cognitivas que requieren de mayor interrelación entre el docente y el alumno (Salas, Romero y Reinoza, 2017).

Por otro lado, los procesos, habilidades y competencias a evaluar en educación inicial, están más relacionados con la experiencia que con los saberes previos y por tanto, difícilmente pueden ser medidos. Los niños en esta etapa obtienen conocimientos dentro de un contexto de diálogo cooperativo y la práctica guiada con tutores experimentados que acompañan la actividad. Estos diálogos los internalizará el niño usándolos posteriormente para regular su propio desempeño, lo cual va a favorecer el desarrollo de las actividades con mayor libertad e independencia. Esto implica la necesidad de considerar y establecer mecanismos de evaluación que resulten acordes a estas necesidades.

Desde los fundamentos Políticos, Técnicos y de Gestión de la política pública establecidos en la Estrategia de Atención Integral a la Primera Infancia De cero a siempre, (2016), "se tiene una concepción de niño y niña como seres sujetos de derecho, comprendidos como seres sociales y singulares y por ende diversos" (p.13), lo cual se convierte en una proclamación importante de resaltar, para establecer unas metas del desarrollo que posibiliten el reconocimiento de cada uno de ellos. Siguiendo la misma línea se pone de manifiesto que:

El desarrollo es un proceso de transformaciones y cambios que no se dan de manera secuencial, acumulativa, lineal, homogénea, e idéntico para todos los niños y las niñas. Este proceso de cambio emerge de la interacción dinámica y continua entre la biología y la experiencia del niño y niña con su medio, propiciando la ampliación de la capacidad reflexiva, reelaborando experiencias, contribuyendo a la capacidad de toma de decisiones, 
favoreciendo a su vez sus sentimientos de autonomía (Estrategia de Atención Integral a la Primera Infancia De cero a siempre, 2016. P.13).

Por esta razón, se establecen unos momentos claves y significativos para hablar de evaluación en la educación inicial, propendiendo entonces a dichas transiciones. De esta manera, un primer momento será la Preconcepción (Prepararse para el abordaje de la maternidad), un segundo momento será la Gestación (Instaurar conocimientos y mecanismos de control fetal), un tercer momento será del nacimiento al primer mes (Adecuación al entorno y a las situaciones), un cuanto momento sería del primer mes de nacido a los 3 años (Manejo de lo colectivo) y un último momento de los 3 a los 6 años (Resolución de situaciones sociales).

En consecuencia, la evaluación en educación inicial debe involucrar por sí sola una serie de elementos determinantes dentro de los procesos de los niños, para comprenderlos de manera integral, entendiendo su transitar por cada uno de los momentos claves que se dan en el desarrollo. Por consiguiente, otro de los conceptos que emergen y merecen el análisis riguroso para considerarlos en la evaluación, son las tan llamadas Áreas del desarrollo, ya que estas se integran de manera sistémica a los logros de aprendizaje que se deben evaluar en los niños. Para el caso que aquí ocupa se constituyen en cuatro Áreas fundamentales: área del desarrollo motor, área del desarrollo cognitivo, área del desarrollo lingüístico y el área del desarrollo social/afectivo.

El Ministerio de Educación Nacional (2009), menciona con relación a las áreas del desarrollo inicial que deben considerarse tres aspectos fundamentales en la concepción de desarrollo. En primer lugar, el desarrollo infantil no es un proceso lineal, sino que presenta avances $y$ retrocesos en las transiciones. En segundo lugar, no se puede definir en el desarrollo un inicio claro, es decir, no inicia desde cero. Y, en tercer lugar, el desarrollo es un proceso que nunca concluye.

Desde estos planteamientos, queda claro que una de las herramientas clave para la evaluación en educación inicial es la observación, en otras palabras, monitorear y controlar el desempeño que cada uno de los niños tiene en los entornos educativos. Desde ahí, se comienza a concebir la evaluación como ese "proceso" que no es aislado, ni propuesto solo por unos momentos, sino que se integra al conocimiento riguroso del niño y vincula otras estrategias que la enriquecen y la dotan de sentido en lo que concierne a la praxis pedagógica.

Por otro lado, en las áreas del desarrollo ninguna prima sobre la otra, todas son igual de importantes para la construcción del sujeto en crecimiento, pero el área social/afectiva, juega un papel determinante en esa instauración del ser, ya que atraviesa todos los procesos de desarrollo. En tal sentido, dentro de los procesos de socialización de los niños, en sus primeras interacciones, se posibilita el entendimiento, los procesos de empatía, solidaridad, justicia, desarrollo moral y el empoderamiento de lo que con el tiempo se denominarán habilidades o competencias blandas.

En otras palabras, no se puede descuidar la función que desempeña el docente desde su rol en las prácticas educativas, pues se debe vincular a ellas como un mediador, un acompañante constante, que conozca y reconozca cada uno de los procesos por los que transitan los niños y proponga estrategias de evaluación de manera individualizada, cualitativa, descriptiva, experiencial y contextualizada teniendo como referente las dimensiones del desarrollo.

Por ende, es preciso pensar la evaluación en educación inicial desde diferentes escenarios, por ejemplo: desde el cuidado, la crianza, la salud, 
la alimentación y nutrición, la puericultura; desde las familias, vinculándolas para que participen de manera activa en los procesos de evaluación de sus hijos, enseñándoles a conocer rutas de atención y de ciudad, estableciendo vínculos directos con ellos y que todo lo que gire en torno al niño, sea pensado de manera consiente por cada uno de los actores y cuidadores que participan de su acompañamiento.

En este sentido, cobra especial relevancia la atención temprana como comprensión de la integración de dichos contextos. Al respecto, García (1995) menciona que:

Pudiera suponerse que los efectos de la atención temprana son el resultado de la interacción entre tipo de centro, características del niño, de la familia y de la personalidad del adulto. Difícilmente puede aislarse el efecto de diferentes tipos de atención temprana cuando en la actualidad, la mayoría de los niños reciben experiencias de diferentes escenarios (centros, padres, abuelos, personal contratado (p.53)

A propósito de la atención temprana, se hace indispensable pensar el niño transversalizado por las cuatro actividades rectoras, ya que estas posibilitan un despliegue del ser social precisamente en la infancia, pues el juego, el arte, la literatura y la exploración del medio, se convierte en el pretexto perfecto para conocer el mundo a través de ellas y se reconocen como actividades propias del ser niño. A partir de ahí, se establece una función social en la escuela, teniendo presente la articulación de la normatización y la normalización que se convierten en elementos de valoración en la evaluación.

De esa manera, es preciso reiterar que las dimensiones del desarrollo en la educación inicial se evalúan todas integradas, a través de proyectos lúdico-pedagógicos, estableciendo relaciones directas en: cómo se enseñó, qué se enseñó y qué se pretende evaluar, teniendo en cuenta los ritmos, necesidades e intereses de los niños, entendiendo también, que para la primera infancia los procesos de socialización son vitales para la construcción del yo. $Y$ de esta manera la socialización se convierte en el encargo social de la escuela y los preescolares.

Finalmente, se hace necesario considerar que la evaluación en el preescolar no tiene calificación cuantitativa, pero sí cualitativa, convirtiéndose de esta manera en un proceso reflexivo y sistémico, que le permite al docente resignificar y valorar al niño en toda su extensión, propendiendo a procesos de enseñanza-aprendizajes pertinentes para la educación inicial.

\section{CONCLUSIONES}

En el presente trabajo se ha realizado un recorrido reflexivo y argumentativo sobre la evaluación en la práctica educativa, actividad que, si bien atraviesa todo el sistema educativo, ha sido objeto de distintas conceptualizaciones y aplicaciones. Al respecto, existen dos grandes tendencias claramente opuestas en relación a la evaluación. Por un lado, la evaluación como medida o estimación numérica, que no solo implica una homogeneización de la concepción del aprendizaje y masificación de los saberes, sino que ha sido punto de partida para la consolidación de sistemas externos de evaluación, los cuales orientan los principios rectores de la calidad educativa.

Por otro lado, se plantea el reto de la evaluación experiencial, en la cual se considera la singularidad, y los distintos estilos y ritmos de aprendizaje. Desde este planteamiento se hace latente la necesidad de desarrollar un entorno de aula fundamentado en el diálogo, la interacción de saberes y la mediación para el logro de aprendizajes significativos. Evidentemente, un modelo evaluativo construido sobre la base de dichas premisas, difícilmente puede considerar 
medidas estandarizadas y generales para valorar el aprendizaje, ya que se considera que la experiencia de aprender es única.

Este segundo enfoque es, si cabe decirlo, un reto importante para los docentes, quienes deben desarrollar mecanismos y estilos didácticos que hagan viable conocer y valorar el aprovechamiento de los objetivos de aprendizaje. En tal sentido, al no considerarse la medición general, se está proponiendo una perspectiva cualitativa con base en las experiencias que emergen del proceso de aprendizaje.

Estas reflexiones permiten poner en la mira los procesos evaluativos utilizados en educación inicial, en los cuales se considera el aprendizaje como una actividad irregular, caracterizada por transiciones, y la interrelación de los distintos contextos de referencia. Es así como la actividad evaluativa en dicha etapa educativa implica un conjunto de competencias, todas ellas interrelacionadas y atravesadas de manera especial por la dimensión socioafectiva como componente clave en todos los ámbitos del desarrollo.

\section{REFERENCIAS BIBLIOGRAFICAS}

Decreto 1290 de Abril 16 de 2009. Por el cual se reglamenta la evaluación del aprendizaje y promoción de los estudiantes de los niveles de educación básica y media. Recuperado de: https://www.mineducacion.gov.co/1759/ w3-article-187765.html? noredirect=1

Delors, J (1996). La educción encierra un tesoro. Informe Unesco de la Comisión de Educación para el siglo XXI. Paris: Ediciones UNESCO. Recuperado de: www. unesco.org/education/pdf/DELORS_S. PDF.

De cero a siempre. (2016). Estrategia de Atención Integral a la Primera Infancia
Fundamentos políticos. Técnicos y de gestión. Presidencia de la República de Colombia

García, M. (1995). La evaluación de la educación infantil. Revista Complutense de Educación. Vol. 6. №1. Pp. 49-72.

Jurado, P. (2009) Calidad de vida y procesos educativos. Revista de Educación Inclusiva, Vol. 2, N² 2. Pp. 1-14. Recuperado de: https://www. revistaeducacioninclusiva.es/index.php/ $\mathrm{REI} /$ article/view/37

Leal Ortiz, N. (2014) La evaluación de los aprendizajes en la UNA desde la perspectiva estudiantil. En: Evaluación educativa en educación a distancia. Experiencias en la Universidad Nacional Abierta de Venezuela. Compilado por María Martín y Leida Sosa L. pp. 27-59. Caracas: Universidad Nacional Abierta; Ediciones del Vicerrectorado Académico.

Ministerio de Educación Nacional. (2009). Desarrollo infantil y competencias en la Primera Infancia. Bogotá: Colombia:

Ministerio de Educación Nacional (2008). Evaluación para los aprendizajes. Altablero No. 44, enero-marzo. Recuperado de: https://www.mineducacion.gov.co/1621/ article-162342.html

OCDE. (2018). PISA results. Recuperado de: http://www.oecd.org/pisa/data/

Salas, M.; Romero, K. y Reinoza, M., (2017) Teorías cognoscitivas. Un recorrido a través de sus principales exponentes. En: Psicología. Aportes a la Educación y al aprendizaje. María Gisela Escobar (coordinadora) pp. 35-46. Mérida: Sello Editorial Universidad de Los Andes. 
Tamayo, L.; Niño, L.; Cardozo, L. y Bejarano,

O. (2017) ¿Hacia dónde va la evaluación? Aportes conceptuales para pensar y transformar las prácticas de evaluación. Bogotá: Instituto para la Investigación Educativa y el Desarrollo Pedagógico 